\title{
An Upwind Solver for the National Combustion Code
}

\author{
Peter M. Sockol \\ NASA Glenn Research Center, Cleveland, Ohio 44135
}

\begin{abstract}
An upwind solver is presented for the unstructured grid National Combustion Code (NCC). The compressible Navier-Stokes equations with time-derivative preconditioning and preconditioned fluxdifference splitting of the inviscid terms are used. First order derivatives are computed on cell faces and used to evaluate the shear stresses and heat fluxes. A new flux limiter uses these same first order derivatives in the evaluation of left and right states used in the flux-difference splitting. The k-epsilon turbulence equations are solved with the same second-order method. The new solver has been installed in a recent version of NCC and the resulting code has been tested successfully in 2D on two laminar cases with known solutions and one turbulent case with experimental data.
\end{abstract}

\section{Introduction}

The National Combustion Code (NCC) is a state of the art Computational Fluiid Dynamics (CFD) program specifically designed for combustion processes ${ }^{1}$. The code employs an unstructured grid, massively parallel computing ${ }^{2,3}$, a dynamic wall function with the effect of adverse pressure gradient ${ }^{4}$, a low Reynolds number wall treatment ${ }^{5}$, a cubic non-linear k-epsilon turbulence model ${ }^{6}$, a lagrangian liquid phase spray $\operatorname{model}^{7}$, and stiff laminar chemistry integration. Recently, viscous low-speed preconditioning ${ }^{8}$ has been added to improve the low-speed convergence of the NCC in viscous regions and the ability to handle multiple sets of periodic boundary conditions has also been added. The combination of these features is usually not available in other CFD codes and gives the NCC an advantage when computing recirculating, turbulent, reacting spray flows. Previously, the NCC has undergone extensive validation studies for simple flows ${ }^{9}$, complex flows ${ }^{10}, \mathrm{NO}_{\mathrm{x}}$ emissions prediction ${ }^{11}$ and traditional gas turbine combustor injectors ${ }^{12}$.

The current NCC solver uses a finite volume discretization with unknowns stored at cell centers. The inviscid fluxes are evaluated with average values on the cell faces and a Jameson-type dissipation operator is added to avoid odd-even decoupling. For the viscous fluxes, first order spatial derivatives at cell centers are obtained by summing cell-face average values and cell-face derivatives are then found by averaging adjacent cell-center values. The equations are advanced in pseudo-time by a four stage RungeKutta scheme in which residual smoothing is applied at each stage. For time-accurate simulations, a dual time-stepping approach is employed.

There are a number of flow regimes in which an upwind solver should produce a more accurate solution than the current NCC solver. The most obvious involve transonic flows with shocks. A less obvious case involves turbulent shear layers where the decrease in artificial dissipation inherent in the upwind method should produce more accurate solutions of the k-epsilon equations. Finally, for reacting flows, the decrease in artificial dissipation should again give more accurate solutions for the species transport equations. In addition, for these flows as well as others, the use of face-centered derivatives in the viscous fluxes should lead to more accurate solutions for all equations.

In the present work, we introduce an upwind solver for use on the unstructured grids of NCC. Preconditioned flux-difference splitting is employed for the inviscid fluxes. First order derivatives are computed on cell faces and used to evaluate shear stresses and heat fluxes. A new flux limiter, based on a nonlinear average of these same face-centered derivatives, is used in the evaluation of the left and right states for flux-difference splitting. Since the test cases in this paper are all two-dimensional non-reacting 
flows, for simplicity the description of the method is similarly limited. The extension to threedimensional reacting flows is straightforward.

\section{Governing Equations}

The governing equations are the two-dimensional compressible Navier-Stokes equations with timederivative preconditioning plus the two-equation $\mathrm{k}-\epsilon$ turbulence model. Let $\mathrm{x}$ and $\mathrm{y}$ be space dimensions, $\mathrm{t}, \mathrm{p}, \mathrm{u}, \mathrm{v}, \mathrm{h}, \mathrm{T}$, and $\rho$ are time, pressure, $\mathrm{x}$ and y velocities, enthalpy, temperature and density, respectively and all variables are dimensional. In addition, for low Mach number flows, it is convenient to subtract off the large background pressure $\bar{p}$ (set by user) by setting

$$
p=\bar{p}+p^{\prime}
$$

The equations are written in vector conservation-law form

$$
P_{c}^{-1} \frac{\partial U}{\partial t}+\frac{\partial}{\partial x}\left(E-E_{v}\right)+\frac{\partial}{\partial y}\left(F-F_{v}\right)=H
$$

Where

$$
\begin{aligned}
& U=\left(\rho, \rho u, \rho v, e^{\prime}, \rho k, \rho \varepsilon\right)^{T}, \\
& E=\left(\rho u, \rho u^{2}+p^{\prime}, \rho u v, \rho u h_{t}, \rho u k, \rho u \varepsilon\right)^{T}, \\
& F=\left(\rho v, \rho u v, \rho v^{2}+p^{\prime}, \rho v h_{t}, \rho v k, \rho v \varepsilon\right)^{T}, \\
& E_{v}=\left(0, \tau_{x x}, \tau_{x y}, u \tau_{x x}+v \tau_{x y}-q_{x}, \tau_{k x}, \tau_{\varepsilon x}\right)^{T}, \\
& F_{v}=\left(0, \tau_{y x}, \tau_{y y}, u \tau_{y x}+v \tau_{y y}-q_{y}, \tau_{k y}, \tau_{\varepsilon y}\right)^{T}, \\
& H=\left(0,0,0,0, S_{k}, S_{\varepsilon}\right)^{T},
\end{aligned}
$$

$P_{c}$ is the preconditioning matrix, $e^{\prime}=\rho h_{t}-p^{\prime}$ is the total energy, and $h_{t}=h+\frac{1}{2}\left(u^{2}+v^{2}\right)$ is the total enthalpy. Note that subtraction of the constant background pressure $\bar{p}$ does not change the basic form of the conservation equations. The density $\rho$ is given by the equation of state for an ideal gas

$$
\rho=\left(\bar{p}+p^{\prime}\right) / R T,
$$

where $R$ is the gas constant for the fluid. The viscous stresses, $k$ and $\varepsilon$ viscous terms and the heat flux vector are given by 


$$
\begin{aligned}
& \tau_{x x}=\mu\left(2 \frac{\partial u}{\partial x}-\frac{2}{3} d i v\right)-\rho \overline{u^{\prime \prime} u^{\prime \prime}}, \quad \tau_{y y}=\mu\left(2 \frac{\partial v}{\partial y}-\frac{2}{3} d i v\right)-\rho \overline{v^{\prime \prime} v^{\prime \prime}}, \\
& \tau_{x y}=\tau_{y x}=\mu\left(\frac{\partial u}{\partial y}+\frac{\partial v}{\partial x}\right)-\rho \overline{u^{\prime \prime} v^{\prime \prime}}, \quad d i v=\frac{\partial u}{\partial x}+\frac{\partial v}{\partial y}, \\
& \tau_{k x}=\left(\mu+\sigma_{k}^{-1} \mu_{t}\right) \frac{\partial k}{\partial x}, \quad \tau_{k y}=\left(\mu+\sigma_{k}^{-1} \mu_{t}\right) \frac{\partial k}{\partial y}, \\
& \tau_{\varepsilon x}=\left(\mu+\sigma_{\varepsilon}^{-1} \mu_{t}\right) \frac{\partial \varepsilon}{\partial x}, \quad \tau_{\varepsilon y}=\left(\mu+\sigma_{\varepsilon}^{-1} \mu_{t}\right) \frac{\partial \varepsilon}{\partial y}, \\
& q_{x}=-\kappa_{\text {eff }} \frac{\partial T}{\partial x}, \quad q_{y}=-\kappa_{e f f} \frac{\partial T}{\partial y},
\end{aligned}
$$

here $\kappa_{\text {eff }}=\kappa+\operatorname{Pr}_{t}^{-1} \mu_{t} C_{p}$ is the effective thermal conductivity, $\mu, \mu_{t}, \kappa$ and $C_{p}$ are the fluid viscosity, eddy viscosity, thermal conductivity and specific heat, respectively. Also $\sigma_{k}^{-1}, \sigma_{\varepsilon}^{-1}$ are model constants and $\operatorname{Pr}_{t}$ is the turbulent Prandtl number. The turbulence source terms $S_{k}, S_{\varepsilon}$ are given by

$$
S_{k}=P_{k}-\rho \varepsilon, \quad S_{\varepsilon}=C_{\varepsilon 1} \frac{\varepsilon}{k} P_{k}-C_{\varepsilon 2} \frac{\rho \varepsilon^{2}}{k},
$$

where the production term $P_{k}$ is

$$
P_{k}=\left(-\overline{\rho u_{i}^{\prime \prime} u_{j}^{\prime \prime}}\right) \frac{\partial u_{i}}{\partial x_{j}},
$$

and $C_{\varepsilon 1}, C_{\varepsilon 2}$ are additional model constants. The Reynolds stresses and the eddy viscosity are given by

$$
\left(-\overline{\rho u_{i}^{\prime \prime} u_{j}^{\prime \prime}}\right)=-\frac{2}{3} \rho k \delta_{i j}+\mu_{t}\left[\left(\frac{\partial u_{i}}{\partial x_{j}}+\frac{\partial u_{j}}{\partial x_{i}}\right)-\frac{2}{3} \frac{\partial u_{k}}{\partial x_{k}} \delta_{i j}\right]
$$

and

$$
\mu_{t}=C_{\mu} \rho \frac{k^{2}}{\varepsilon} .
$$

Note that we are using only the linear terms in the Reynolds stress model ${ }^{6}$. However, we have found it necessary to use the variable $C_{\mu}$ from that reference in order to capture the near wall behavior. This is given by

$$
\begin{aligned}
& C_{\mu}=\frac{1}{4+A_{S} U^{*}(k / \varepsilon)}, \\
& U^{*}=\sqrt{\left(S^{*}\right)^{2}+\left(\Omega^{*}\right)^{2}}, \quad S^{*}=\sqrt{S_{i j}^{*} S_{i j}^{*}}, \quad \Omega^{*}=\sqrt{\Omega_{i j} \Omega_{i j}}, \quad \Omega_{i j}=\frac{1}{2}\left(\frac{\partial u_{i}}{\partial x_{j}}-\frac{\partial u_{j}}{\partial x_{i}}\right), \\
& S_{i j}^{*}=S_{i j}-\frac{1}{3} S_{k k} \delta_{i j}, \quad S_{i j}=\frac{1}{2}\left(\frac{\partial u_{i}}{\partial x_{j}}+\frac{\partial u_{j}}{\partial x_{i}}\right), \\
& A_{S}=\sqrt{6} \cos \phi, \quad \phi=\frac{1}{3} \cos ^{-1}\left(\sqrt{6} W^{*}\right), \quad W^{*}=\frac{S_{i j}^{*} S_{j k}^{*} S_{k i}^{*}}{\left(S^{*}\right)^{3}} .
\end{aligned}
$$


For turbulent flows the generalized wall function ${ }^{4}$ is applied at all solid walls.

The preconditioning matrix $P_{c}$ is adapted from the work of Turkel ${ }^{13}$. First consider the inviscid equations written in terms of the primitive variables $V=\left(p^{\prime}, u, v, S^{\prime}, k, \varepsilon\right)^{T}$, where $d S^{\prime}=d p^{\prime}-c^{2} d \rho$, $c^{2}=\gamma R T$ is the speed of sound and $\gamma$ is the ratio of specific heats. These are written as

$$
P_{p}^{-1} \frac{\partial V}{\partial t}+A_{p} \frac{\partial V}{\partial x}+B_{p} \frac{\partial V}{\partial y}=0
$$

with

$$
A_{p}=\left[\begin{array}{cccccc}
u & \rho c^{2} & 0 & 0 & 0 & 0 \\
\rho^{-1} & u & 0 & 0 & 0 & 0 \\
0 & 0 & u & 0 & 0 & 0 \\
0 & 0 & 0 & u & 0 & 0 \\
0 & 0 & 0 & 0 & u & 0 \\
0 & 0 & 0 & 0 & 0 & 0
\end{array}\right], \quad B_{p}=\left[\begin{array}{cccccc}
v & 0 & \rho c^{2} & 0 & 0 & 0 \\
0 & v & 0 & 0 & 0 & 0 \\
\rho^{-1} & 0 & v & 0 & 0 & 0 \\
0 & 0 & 0 & v & 0 & 0 \\
0 & 0 & 0 & 0 & v & 0 \\
0 & 0 & 0 & 0 & 0 & v
\end{array}\right] .
$$

For this system the simplest form of Turkel's preconditioner is a diagonal matrix written as

$$
P_{p}^{-1}=\operatorname{Diag}\left(m^{-2}, 1,1,1,1,1\right)
$$

where $m^{2}=\beta^{2} / c^{2}$ and $\beta^{2}$ is chosen to bring the system eigenvalues closer together at low Mach numbers. The eigenvalues of $P_{p} A_{p}$ are given by

$$
\lambda=u-u_{0}-c_{0}, u, u, u, u, u-u_{0}+c_{0}
$$

with

$$
c_{0}^{2}=u_{0}^{2}+\beta^{2}, \quad u_{0}=\frac{1}{2}\left(1-m^{2}\right) u .
$$

The conservation-law form is then given by $P_{c}=M P_{p} M^{-1}$ with $M=\partial U / \partial V$. Finally, following Choi and Merkle ${ }^{8}$, we express Eq.(2) in terms of altered primitive variables $Q=\left(p^{\prime}, u, v, h, k, \varepsilon\right)^{T}$

$$
P_{Q}^{-1} \frac{\partial Q}{\partial t}+\frac{\partial}{\partial x}\left(E-E_{v}\right)+\frac{\partial}{\partial y}\left(F-F_{v}\right)=H
$$

where $P_{Q}^{-1}=M P_{p}^{-1} N$ and $N=\partial V / \partial Q$.

For most flows it is sufficient to set $\beta^{2}$ equal to the square of the local flow speed together with lower and upper limits. However, for viscous flows near walls, convergence is improved by introducing the viscous limit of Choi and Merkle ${ }^{8}$. Hence we take

$$
\beta^{2}=\min \left[\max \left(\beta_{0}^{2}, u^{2}+v^{2}, \beta_{v}^{2}\right), c^{2}\right]
$$

where $\beta_{0}^{2}$ is a global user specified lower limit and 


$$
\beta_{v}^{2}=\frac{\alpha(\alpha-1) v_{n}^{2}}{1+(\alpha-1) v_{n}^{2} / c^{2}} \text {. }
$$

Here $\alpha=R C V / \operatorname{Re}_{\text {cell }}, R C V$ is the ratio of CFL to von Neumann numbers (user specified), $\operatorname{Re}_{\text {cell }}=\rho v_{n} \delta_{n} / \mu_{\text {eff }}$ is the local cell Reynolds number, $v_{n}$ is the velocity normal to the cell face and $\delta_{n}$ is the spacing between adjacent cell centers.

\section{Discrete Formulation}

The governing equations are solved on an unstructured grid (triangles or quads in 2D) with unknowns stored at cell centers. Integration of Eq. (16) over a cell together with explicit time differencing gives

$$
P_{Q}^{-1}(\Delta A / \Delta t) \Delta Q=-R^{n}
$$

where $\Delta A$ is the cell area, $\Delta t$ is the time step, $\Delta Q=Q^{n+1}-Q^{n}$, and the area-weighted residual $R$ is given by

$$
R_{i}=\sum_{j}\left\lfloor\left(E-E_{v}\right)_{i j} d y_{i j}-\left(F-F_{v}\right)_{i j} d x_{i j}\right\rfloor-H_{i} \Delta A_{i},
$$

where the summation is over all faces $j$ of cell $i$, while $d y_{i j}, d x_{i j}$ are the changes in $y, x$ respectively, along face $j$.

The cell-face inviscid fluxes $E$ and $F$ in Eq. (20) are approximated by preconditioned fluxdifference splitting ${ }^{14}$

$$
E=\frac{1}{2}\left[E\left(U^{L}\right)+E\left(U^{R}\right)\right]-\frac{1}{2} \hat{P}_{c}^{-1}\left|\hat{P}_{c} \hat{A}_{c}\right|\left(U^{R}-U^{L}\right),
$$

where $U^{L}$ and $U^{R}$ are, respectively, left and right states, $A_{c}=\partial E / \partial U, \hat{A}_{c}$ denotes evaluation at an average state, and $P_{c}^{-1}\left|P_{c} A_{c}\right|=M P_{p}^{-1}\left|P_{p} A_{p}\right| M^{-1}$. Here $\left|P_{p} A_{p}\right|$ is computed using the eigenvectors and absolute eigenvalues of $P_{p} A_{p}$. Eq.(21) is rewritten in terms of the altered primitive variables $Q$

$$
E=\frac{1}{2}\left[E\left(Q^{L}\right)+E\left(Q^{R}\right)\right]-\frac{1}{2} \hat{A}^{a}\left(Q^{R}-Q^{L}\right),
$$

where $A^{a}=M P_{p}^{-1}\left|P_{p} A_{p}\right| N$. The left and right states $Q^{L}, Q^{R}$ are obtained by extrapolating from the adjacent cell-centers. Note this linear extrapolation leads to a second order approximation for the fluxes. Let $i 1, i 2$ denote the centers of two adjacent cells and $f_{c}$ denote the centroid of the cell face separating these two cells. Then

$$
\begin{aligned}
& Q^{L}=Q_{i 1}+\left(\frac{\partial Q}{\partial x}\right)_{i 1}\left(x_{f c}-x_{i 1}\right)+\left(\frac{\partial Q}{\partial y}\right)_{i 1}\left(y_{f c}-y_{i 1}\right), \\
& Q^{R}=Q_{i 2}+\left(\frac{\partial Q}{\partial x}\right)_{i 2}\left(x_{f c}-x_{i 2}\right)+\left(\frac{\partial Q}{\partial y}\right)_{i 2}\left(y_{f c}-y_{i 2}\right),
\end{aligned}
$$

In the present implementation the average state $\hat{Q}$ is taken as a simple average of $Q^{L}$ and $Q^{R}$. The flux $F$ is approximated by expressions similar to Eqs. (21) and (22) with $A_{c}$ replaced by $B_{c}=\partial F / \partial U$ and $A^{a}$ replaced by $B^{a}=M P_{p}^{-1}\left|P_{p} B_{p}\right| N$.

The cell-center derivatives in Eq. (23) are obtained by a weighted average process that is a modification of that used by Hunnh ${ }^{15}$ for triangular cells. Let $i j$ represent face $j$ of cell $i$ and set 


$$
\theta_{i j}=\left(\frac{\partial Q}{\partial x}\right)_{i j}^{2}+\left(\frac{\partial Q}{\partial y}\right)_{i j}^{2}, \quad w t_{i j}=\prod_{k \neq j} \theta_{i k}, \quad d n=\sum_{j} w t_{i j},
$$

then

$$
\left(\frac{\partial Q}{\partial x}\right)_{i}=d n^{-1} \sum_{j} w t_{i j}\left(\frac{\partial Q}{\partial x}\right)_{i j}, \quad\left(\frac{\partial Q}{\partial y}\right)_{i}=d n^{-1} \sum_{j} w t_{i j}\left(\frac{\partial Q}{\partial y}\right)_{i j} .
$$

where these expressions are applied to each of the variables in $Q$. Note the resulting cell-center derivative is biased toward the least steep of the surrounding cell-face slopes and thus serves as a flux limiter. Also, this formulation works for any cell shape, in two or three dimensions.

The cell-face derivatives are obtained by integrating over a volume surrounding the face. Let $i 1, i 2$ denote the centers of two adjacent cells and $k 1, k 2$ the ends of the face separating these two cells. Averaging the derivatives over this volume gives

$$
\begin{aligned}
& \left(\frac{\partial Q}{\partial x}\right)_{f}=\frac{1}{2} d A_{f}^{-1}\left[\left(Q_{i 2}-Q_{i 1}\right) d y^{k}-\left(Q_{k 2}-Q_{k 1}\right) d y^{i}\right] \\
& \left(\frac{\partial Q}{\partial y}\right)_{f}=-\frac{1}{2} d A_{f}^{-1}\left[\left(Q_{i 2}-Q_{i 1}\right) d x^{k}-\left(Q_{k 2}-Q_{k 1}\right) d x^{i}\right]
\end{aligned}
$$

where

$$
\begin{aligned}
& d x^{i}=x_{i 2}-x_{i 1}, \quad d y^{i}=y_{i 2}-y_{i 1}, \quad d x^{k}=x_{k 2}-x_{k 1}, d y^{k}=y_{k 2}-y_{k 1}, \\
& d A_{f}=\frac{1}{2}\left(d x^{i} d y^{k}-d y^{i} d x^{k}\right) .
\end{aligned}
$$

Since $Q_{k 1}$ and $Q_{k 2}$ are not stored on the grid, their difference has to be approximated. First we obtain simple approximations to the cell-center derivatives by integrating over the cell volumes,

$$
\left(\frac{\partial Q}{\partial x}\right)_{i}=d A_{i}^{-1} \sum_{j} Q_{j} d y^{j}, \quad\left(\frac{\partial Q}{\partial y}\right)_{i}=-d A_{i}^{-1} \sum_{j} Q_{j} d x^{j}
$$

where the sum is over all the cell faces and the face values $Q_{j}$ are taken as the average of the adjacent cell-center values. Then we approximate $Q_{k 2}-Q_{k 1}$ as

$$
Q_{k 2}-Q_{k 1} \approx \frac{1}{2}\left[\left(\frac{\partial Q}{\partial x}\right)_{i 1}+\left(\frac{\partial Q}{\partial x}\right)_{i 2}\right] d x^{k}+\frac{1}{2}\left[\left(\frac{\partial Q}{\partial y}\right)_{i 1}+\left(\frac{\partial Q}{\partial y}\right)_{i 2}\right] d y^{k}
$$

Eqs. (26) together with Eq. (29) are used to evaluate the cell-face derivatives. These are used not only for the weighted averages of Eq. (22) but also for the cell-face shear stresses and heat fluxes of Eq. (5).

\section{Time Marching Solution}

Eq.(19) for $Q$ is advanced in pseudo-time by a four stage Runge-Kutta scheme. The viscous stresses and heat transfer in Eq. (5) together with the numerical dissipation in Eq. (22) are updated in the first two stages only. For the turbulence variables $k$ and $\varepsilon$, the updated values are under-relaxed at each RungeKutta stage. Finally, residual smoothing is applied at each stage using two sweeps of an under-relaxed Jacobi iteration. 
For the steady flow problems considered here, convergence is based on the root-mean-square of the

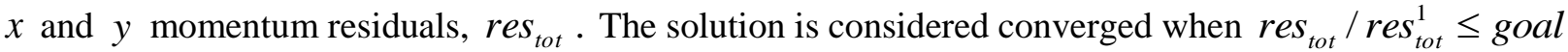
for 100 consecutive iterations, where res $_{\text {tot }}^{1}$ is the value after 1 iteration and goal $=5 \times 10^{-4}$ for the two laminar cases and goal $=10^{-3}$ for the turbulent case. For all cases the CFL number is set to 4 while the Von Neumann number is set to 1 for the laminar cases and 0.5 for the turbulent case.

\section{Results and Discussion}

Three test cases have been chosen to demonstrate the accuracy of the upwind method. For the two laminar cases, existing numerical results are used to check the current solutions. For the turbulent case the present results are compared with experimental data. Note, for the laminar cases, we assume air at $1 \mathrm{~atm}$. and $300 \mathrm{~K}$ with a reference length $D$ of $1 \mathrm{~m}$. The reference velocity $u_{\text {ref }}$ is then chosen to give the desired Reynolds number, $\operatorname{Re}=\rho u_{r e f} D / \mu$.

\section{Lid-driven cavity}

The lid-driven cavity is a common benchmark for recirculating flows. The second-order streamfunction-vorticity results of Ghia et $\mathrm{al}^{16}$., computed on a uniform 256 by 256 grid, are generally accepted as standard. Isothermal flow is set up in a square cavity, side 1, with a top that moves to the right at constant speed $u_{r e f}$. The current work uses a triangular grid with 128 cells along each wall and grid spacing along the wall varying from 0.0005 in the corners to 0.02 in the middle. The resulting grid, with 10,514 triangular cells, is shown in Fig. 1.

The case $\operatorname{Re}=1000$ is obtained by setting $u_{r e f}=0.015936 \mathrm{~m} / \mathrm{sec}$. A contour plot for $u / u_{r e f}$, shown in Fig. 2, gives the general structure of the flow. Note the two recirculating regions in the bottom corners of the flow. Convergence to the desired level is shown in Fig. 3. Note that this took 6683 iterations. Profiles of $u / u_{r e f}$ on the vertical centerline and $v / u_{\text {ref }}$ on the horizontal centerline are compared with the Ghia et al. results in Figs. 4 and 5, respectively. Considering the sparseness of the grid, the comparison is excellent.

\section{Laminar backward-facing step}

We consider isothermal laminar flow over a backward-facing step with a step height of half the downstream channel height $D$ and a parabolic inlet velocity $u(y)=24 y(0.5-y) u_{\text {ref }}$ for $0 \leq y \leq 0.5$ specified at the step. The outflow boundary is taken at 15 channel heights downstream of the step. Gartling ${ }^{17}$ set up this problem as a test for outflow boundary conditions. Numerical results were obtained for $\operatorname{Re}=800$ with the outflow boundary at 30 channel heights and used a Galerkin-based finite element method with $800 \times 40$ biquadratic velocity elements and linear discontinuous pressure elements. The current work uses a 240 by 64 quad grid with stretching in $y$ only. The grid spacing is set at 0.001 at the top and bottom walls and 0.03 at the centerline.

The case $\operatorname{Re}=800$ is given by setting $u_{r e f}=0.012749 \mathrm{~m} / \mathrm{sec}$. A contour plot for $u / u_{\text {ref }}$, shown in Fig. 6, gives the structure of the flow. Note the separation bubble on the upper wall at $x \approx 7$. Convergence to the desired level is shown in Fig. 7. Profiles of $u / u_{r e f}$ at $x=7$ and $x=15$ are compared with Gartling's results in Figs. 8 and 9, respectively. Again the results are quite good. Note that $x=15$ is the exit boundary for the current computation. 


\section{Turbulent backward-facing step}

Kim, Kline and Johnston ${ }^{18,19}(\mathrm{KKJ})$ studied the turbulent flow over a backward-facing step with two main objectives. The first was to characterize the flow in the separated shear layer, the reattachment region, and the developing boundary layer downstream of reattachment. The second was to obtain experimental data that would be useful in the development of computational models. In KKJ's experiment the step height $h$ was $3.81 \mathrm{~cm}$, the channel width upstream of the step was $2 h$, and boundary layer trips were placed on each side wall at a distance of $8 h$ upstream of the step. On the centerline of the channel, a distance of $4 h$ upstream of the step (termed the reference point), the reference speed $u_{r e f}$ was $18.2 \mathrm{~m} / \mathrm{s}$ and the dynamic pressure was $20.3 \mathrm{~mm}$ of water.

In the current work the computational domain is taken from $8 h$ upstream to $30 h$ downstream of the step. We use a stretched quad grid with 30 by 32 cells $(x, y)$ upstream and 50 by 64 cells downstream. The $y$ grid spacing is set at $0.002 h$ along both side walls. The $x$ grid spacing varies from $0.1 h$ at the upstream boundary $(-8 h)$ to $0.4 h$ at the step, remains constant to $x=10 h$, and then increases gradually to $1.312 h$ at the exit $(30 h)$. The resulting grid, from $x / h=-4$ to 20 , is shown in Fig. 10 . The computation is started at $-8 h$ to allow the turbulent boundary layers to grow to suitable thickness before reaching the reference point. At the upstream boundary we assume a uniform inflow with a speed $u_{i n}$, turbulence level of $5 \%$, and a mixing length of $0.001 \mathrm{~m}$. The inlet speed was varied until the solution at the reference point agreed with the experimental value $u_{\text {ref }}=18.2 \mathrm{~m} / \mathrm{sec}$. This required a value of $u_{\text {in }}=$ $17.5 \mathrm{~m} / \mathrm{sec}$. Note this value was not very sensitive to the inlet values of turbulence level and mixing length. A contour plot of $u / u_{\text {ref }}$, shown in Fig. 11, gives the general structure of the flow and convergence to the desired level is shown in Fig. 12. The computed reattachment length, as determined from the change in sign of the wall shear stress, was $x_{R} / h=6.75$. This is well within the experimental range of $7 \pm 1$ reported by KKJ.

The computed wall-static pressure on the step side of the channel (plotted as the pressure coefficient $C p$ ) is compared with experimental values in Fig. 13. Here $C p$ is given by

$$
C p=\frac{p-p_{r e f}}{\frac{1}{2} \rho u_{r e f}^{2}} .
$$

We determine $p_{\text {ref }}$ by forcing the computed pressure to go through the first data point downstream of the step. This comparison is quite good although the computed pressure rise occurs a little early. Profiles of

$u / u_{\text {ref }}$ at four locations downstream of the step are compared with the experimental values in Figs. 14 to 17. Again the comparison is quite good except in the reverse flow portion of the separation zone. Here KKJ note that the flow was unsteady and the measurements may not be accurate.

\section{Conclusions}

An upwind solution method for the unstructured grid National Combustion Code has been presented. The solver uses preconditioned flux-difference splitting for the inviscid terms and face-centered first derivatives for the shear stresses and heat fluxes. A new flux limiter uses these same first order derivatives in the evaluation of left and right states used in the flux-difference splitting. The same methodology is used for the k-epsilon turbulence equations. The solver has been tested in 2D on two laminar and one turbulent flow and shown robust convergence in all cases. The laminar results appear quite accurate when compared with known computational solutions. The turbulent results are in good 
agreement with the experimental data. The second order solution of the k-epsilon equations shows promise for the future analysis of turbulent reacting flows.

\section{References}

${ }^{1}$ Stubbs, R. M. and Liu, N.-S., (1997), "Preview of the National Combustion Code", AIAA-97-3114, 33 ${ }^{\text {rd }}$ AIAA/ASME/SAE/ASEE Joint Propulsion Conference \& Exhibit, July 6-9, Seattle, WA, USA.

${ }^{2}$ Quealy, A., Ryder, R., Norris, A. and Liu, N.-S., (2000), "National Combustion Code: Parallel Implementation and Performance", NASA TM-2000-209801.

${ }^{3}$ Quealy, A., (2002), "National Combustion Code Parallel Performance Enhancements", NASA CR2002-211340.

${ }^{4}$ Shih, T.-H., Povinelli, L. A. and Liu N.-S., (2003), "Application of Generalized Wall Function for Complex Turbulent Flows", Journal of Turbulence, Vol. 4, No. 15, pp 1-16.

${ }^{5}$ Chien, K. Y., (1982), "Prediction of Boundary Layer Flows with a Low-Reynolds-Number Turbulence Model”, AIAA J., Vol. 20, No.1, pp 33-38.

${ }^{6}$ Shih, T.-H., Chen, K.-H. and Liu, N.-S., (1998), "A Non-Linear k-epsilon Model for Turbulent Shear Flows", AIAA-98-3983, 34 ${ }^{\text {th }}$ AIAA/ASME/SAE/ASEE Joint Propulsion Conference \& Exhibit, July 1315, Cleveland, OH, USA.

${ }^{7}$ Raju, M. S., (2004), "LSPRAY-II: A Lagrangian Spray Module”, NASA CR-2004-212958.

${ }^{8}$ Choi, Y.-H. and Merkle, C. L., (1993), “The Application of Preconditioning in Viscous Flows”, J. Comput. Phys., Vol. 105, pp 207-223.

${ }^{9}$ Chen, K.-H., Norris, A. T., Quealy, A. and Liu, N.-S., (1998), "Benchmark Test Cases for The National Combustion Code", AIAA-98-3855, 34 ${ }^{\text {th }}$ AIAA/ASME/SAE/ASEE Joint Propulsion Conference \& Exhibit, July 13-15, Cleveland, OH, USA.

${ }^{10}$ Iannetti, A.,Tacina, R., Jeng, S.-M. and Cai, J., (2001), "Towards Accurate Prediction of Turbulent, Three-Dimensional, Recirculating Flows with the NCC", NASA TM-2001-210761, AIAA-2001-0809.

${ }^{11}$ Shih, T.-H., Norris, A., Iannetti, A. C., Marek, C. J., Smith, T. D., Liu, N.-S. and Povinelli, L. A., (2001), "A Study of Hydrogen/Air Combustor Using NCC", AIAA-2001-808, $39^{\text {th }}$ Aerospace Sciences Meeting \& Exhibit, Jan. 8-11, Reno, NV, USA.

${ }^{12}$ Iannetti, A. C. and Chen, K.-H., (2000), “An Initial Comparison of National Combustion Code Simulations Using Various Chemistry Modules with Experimental Gas Turbine Combustor Data", AIAA2000-0330, $38^{\text {th }}$ Aerospace Sciences Meeting, Reno, NV, USA.

${ }^{13}$ Turkel, E., (1987), "Preconditioned Methods for Solving the Incompressible and Low Speed Compressible Equations”, J. Comput. Phys., Vol. 72, pp 277-298.

${ }^{14}$ van Leer, B., Lee, T. W. and Roe, P., (1991), "Characteristic Time-Stepping or Local Preconditioning of the Euler Equations", AIAA paper 91-1552-CP.

${ }^{15}$ Huynh, H. T., (2003), "Analysis and Improvement of Upwind and Centered Schemes on Quadrilateral and Triangular Meshes", AIAA-2003-3541, $16^{\text {th }}$ AIAA Computational Fluid Dynamics Conference and the $33^{\text {rd }}$ Fluid Dynamics Conference and Exhibit, June 23-26, Orlando, FL.

${ }^{16}$ Ghia, U., Ghia, K. N. and Shin, C. T., (1982), "High-Re Solutions for Incompressible Flow Using the Navier-Stokes Equations and a Multigrid Method”, J. Comput. Phys., Vol. 48, pp 387-411.

${ }^{17}$ Gartling, D. K., (1990), “ A Test Problem for Outflow Boundary Conditions - Flow Over a BackwardFacing Step”, Int. J. Numer. Methods Fluids, Vol. 11, pp 953-967.

${ }^{18}$ Kim, J., Kline, S. J. and Johnston, J. P., (1978), "Investigation of a Reattaching Turbulent Shear Layer: Flow Over a Backward-Facing Step”, MD-37, Thermosciences Division, Dept. of Mechanical Engineering, Stanford University.

${ }^{19}$ Kim, J., Kline, S. J. and Johnston, J. P., (1980), "Investigation of a Reattaching Turbulent Shear Layer: Flow Over a Backward-Facing Step”, J. Fluids Eng., Vol. 102, pp 302-308. 


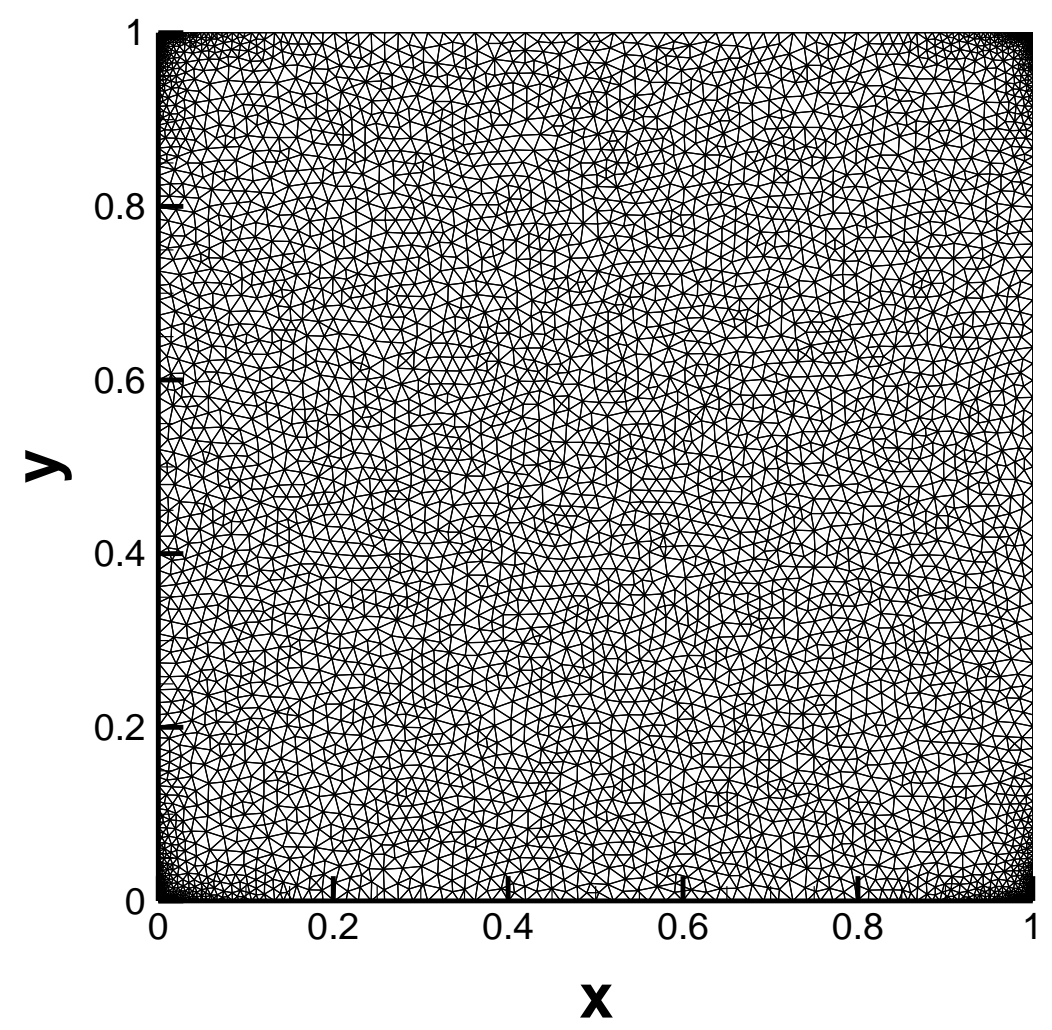

Fig. 1. Grid for lid-driven cavity with 10,514 triangular cells.

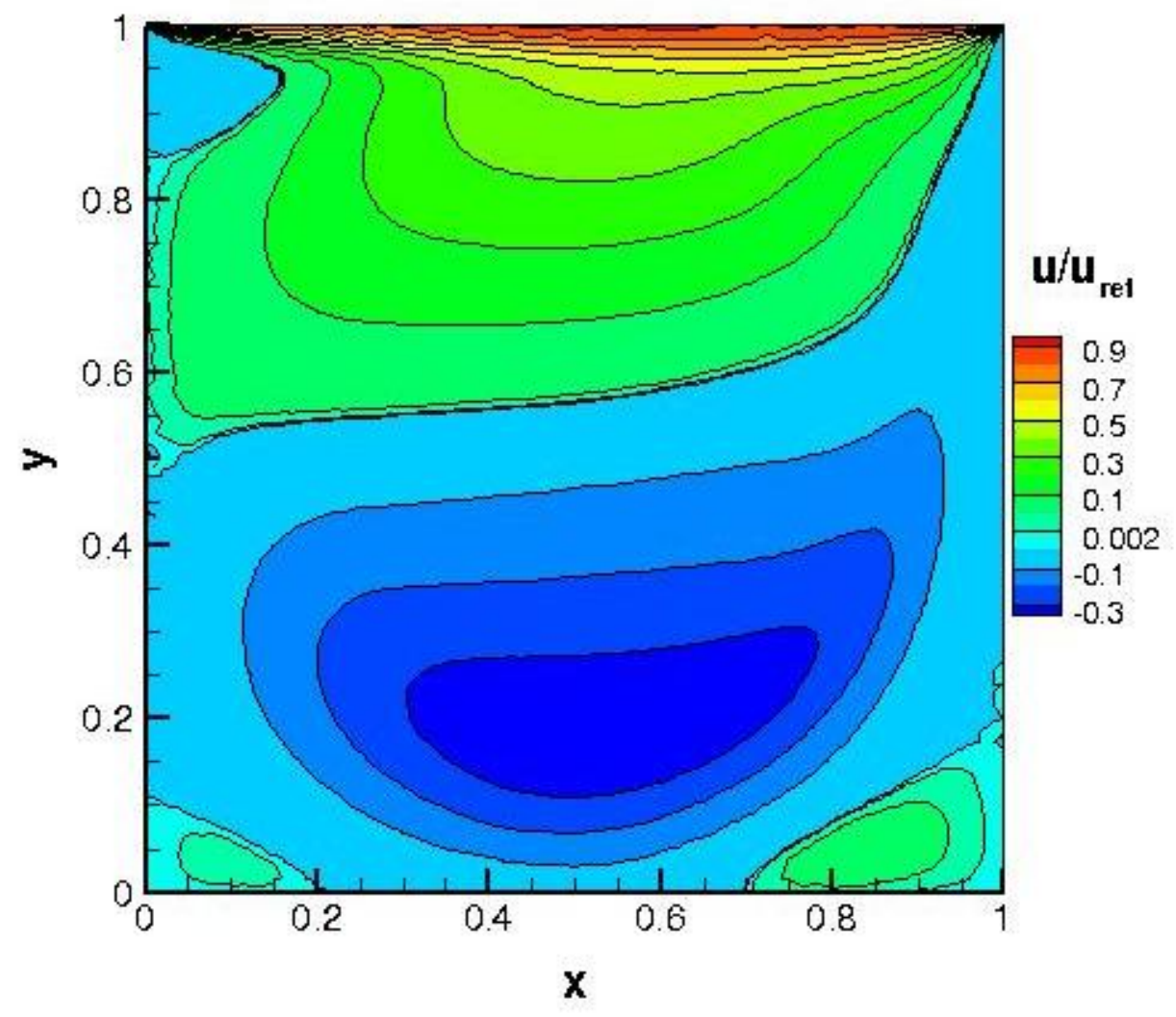

Fig. 2. Contours of $\mathbf{u} / \mathbf{u}_{\text {ref }}$ for the lid-driven cavity at $\mathrm{Re}=1000$. 


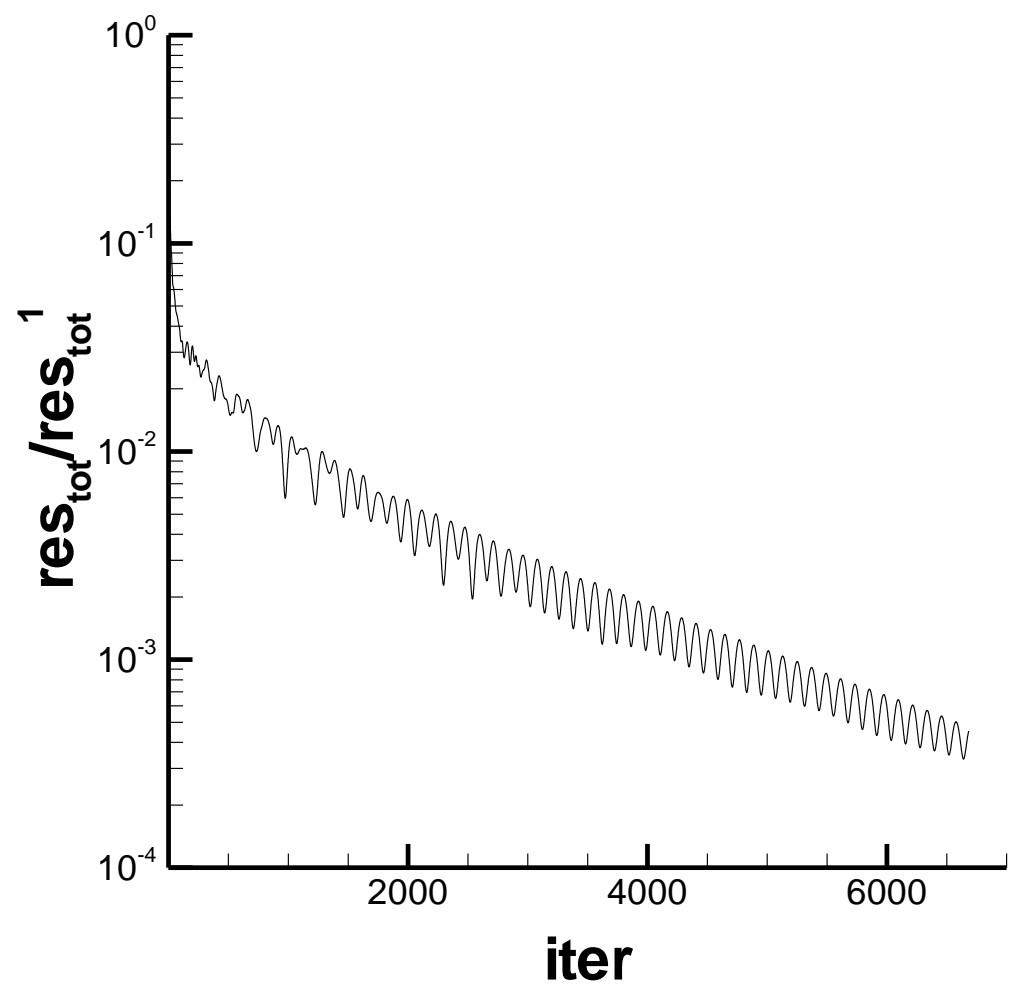

Fig. 3. Convergence of $\mathbf{r e s}_{\text {tot }} / \mathbf{r e s}_{\text {tot }}{ }^{1}$ for the lid-driven cavity at $\mathrm{Re}=1000$.

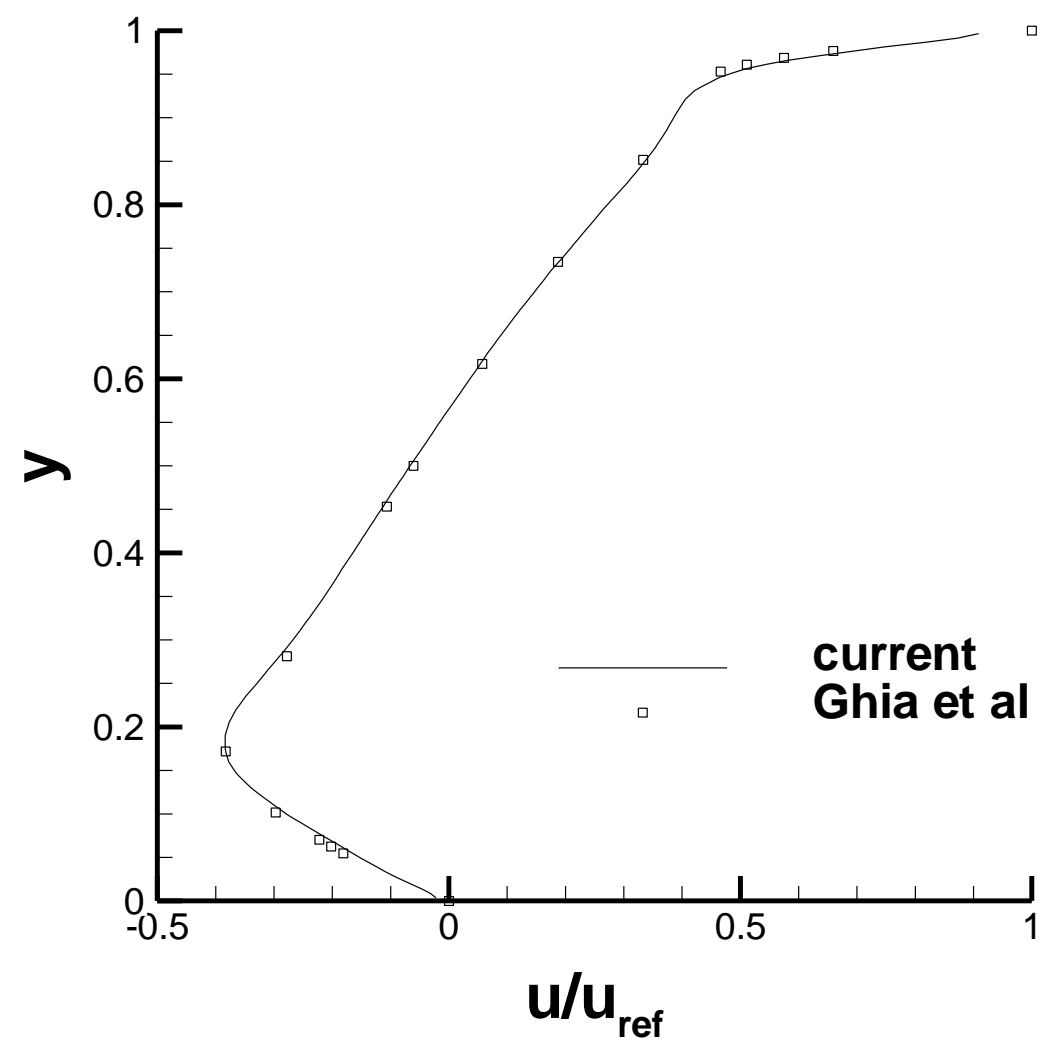

Fig. 4. Comparison of $\mathbf{u} / \mathbf{u}_{\text {ref }}$ on the vertical centerline with the results of Ghia et al for $\operatorname{Re}=1000$. 


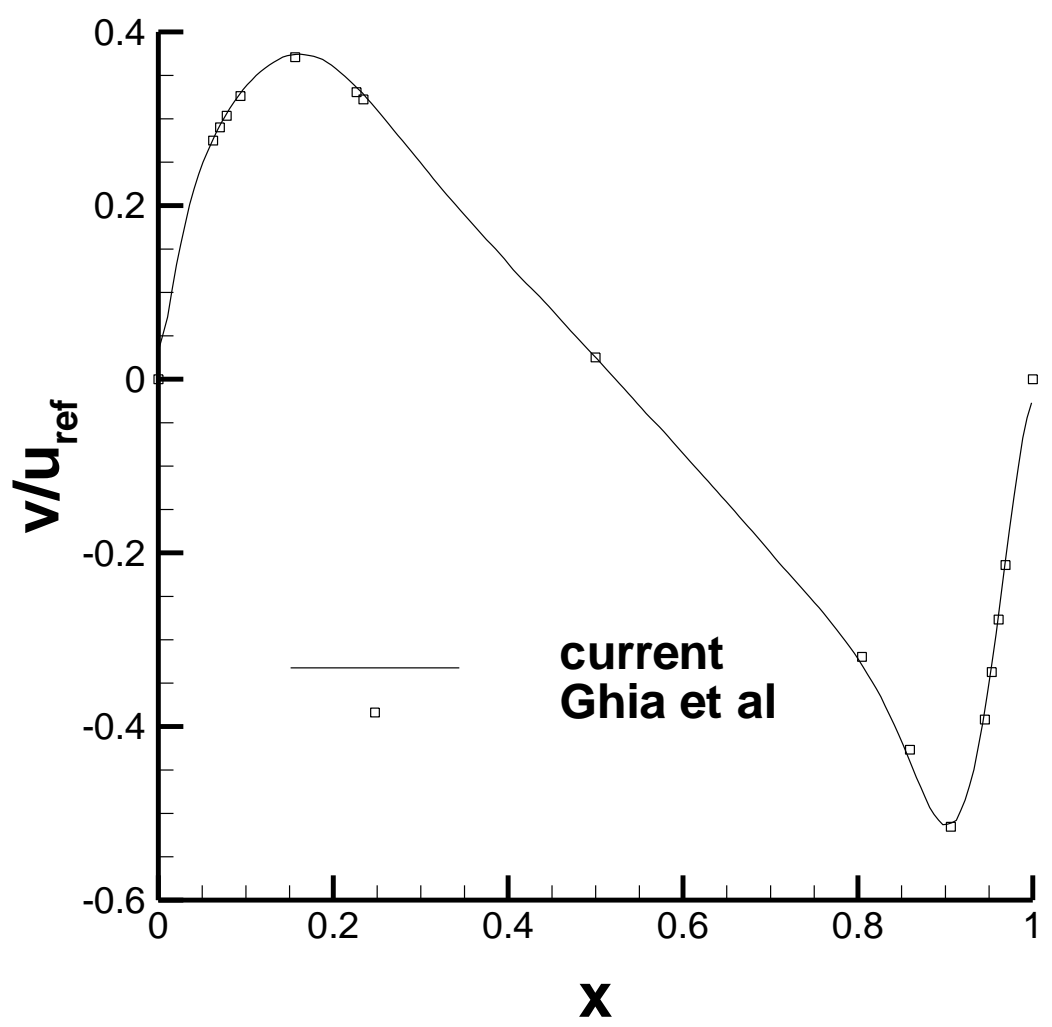

Fig. 5. Comparison of $\mathbf{v} / \mathbf{u}_{\text {ref }}$ on the horizontal centerline with the results of Ghia et al for $\operatorname{Re}=1000$.
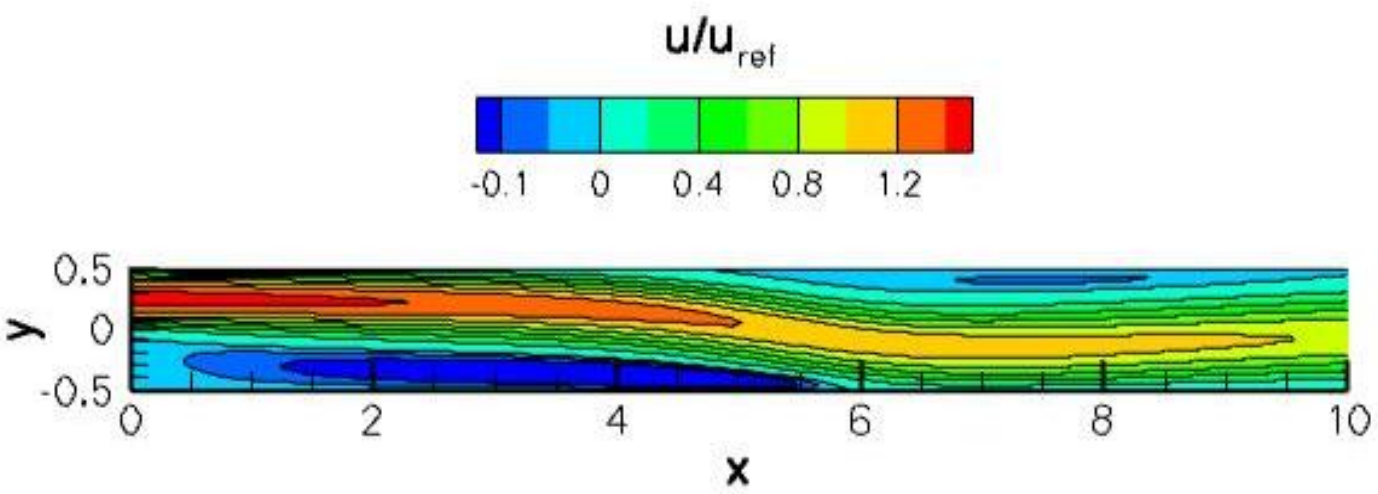

Fig. 6. Contours of $\mathbf{u} / \mathbf{u}_{\text {ref }}$ for the laminar backward-facing step at $\operatorname{Re}=800$, (where the grid portion from $\mathrm{x}=10$ to 15 is not shown). 


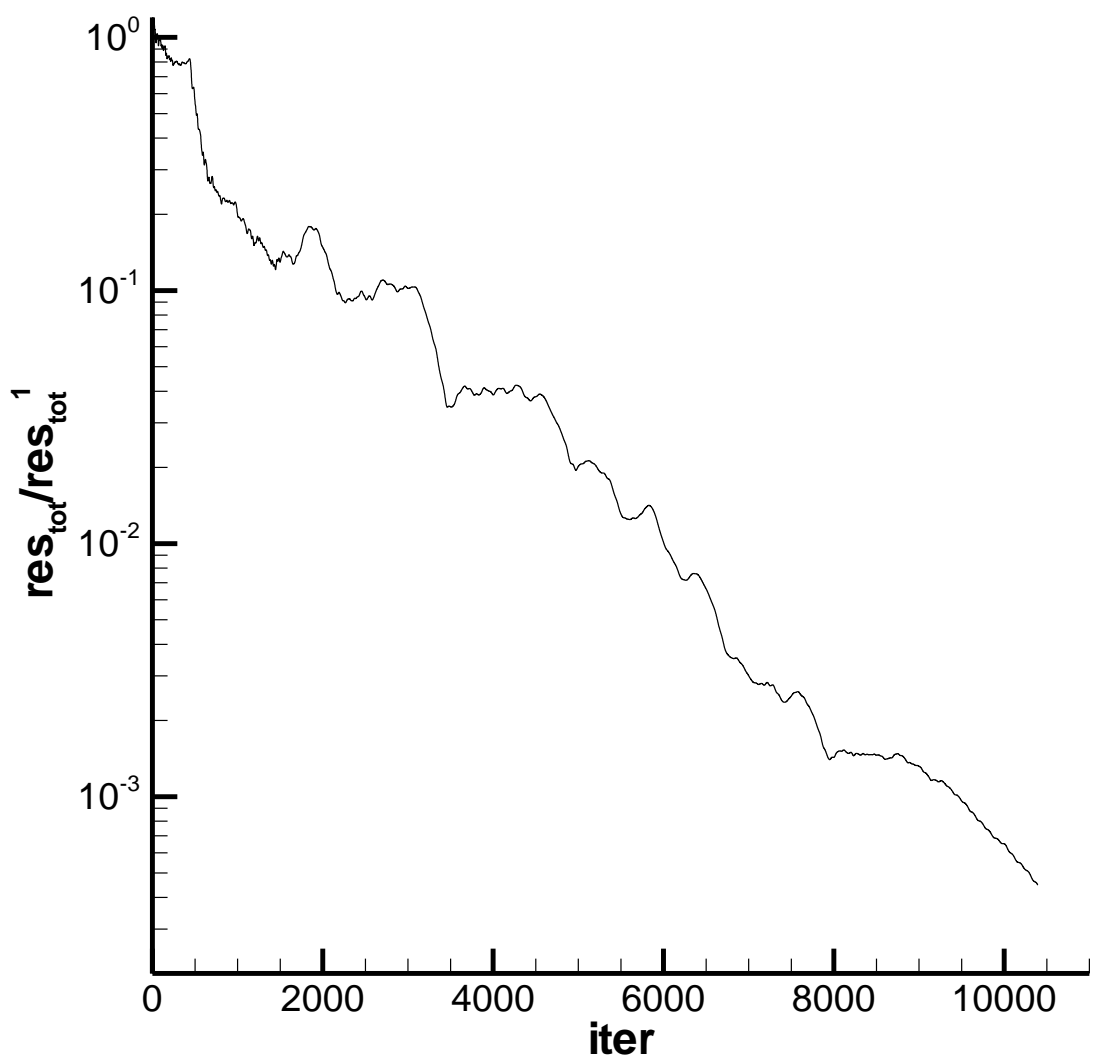

Fig. 7. Convergence of $\mathbf{r e s}_{\text {tot }} / \mathbf{r e s}_{\text {tot }}{ }^{1}$ for the laminar backward-facing step at $\mathrm{Re}=800$.

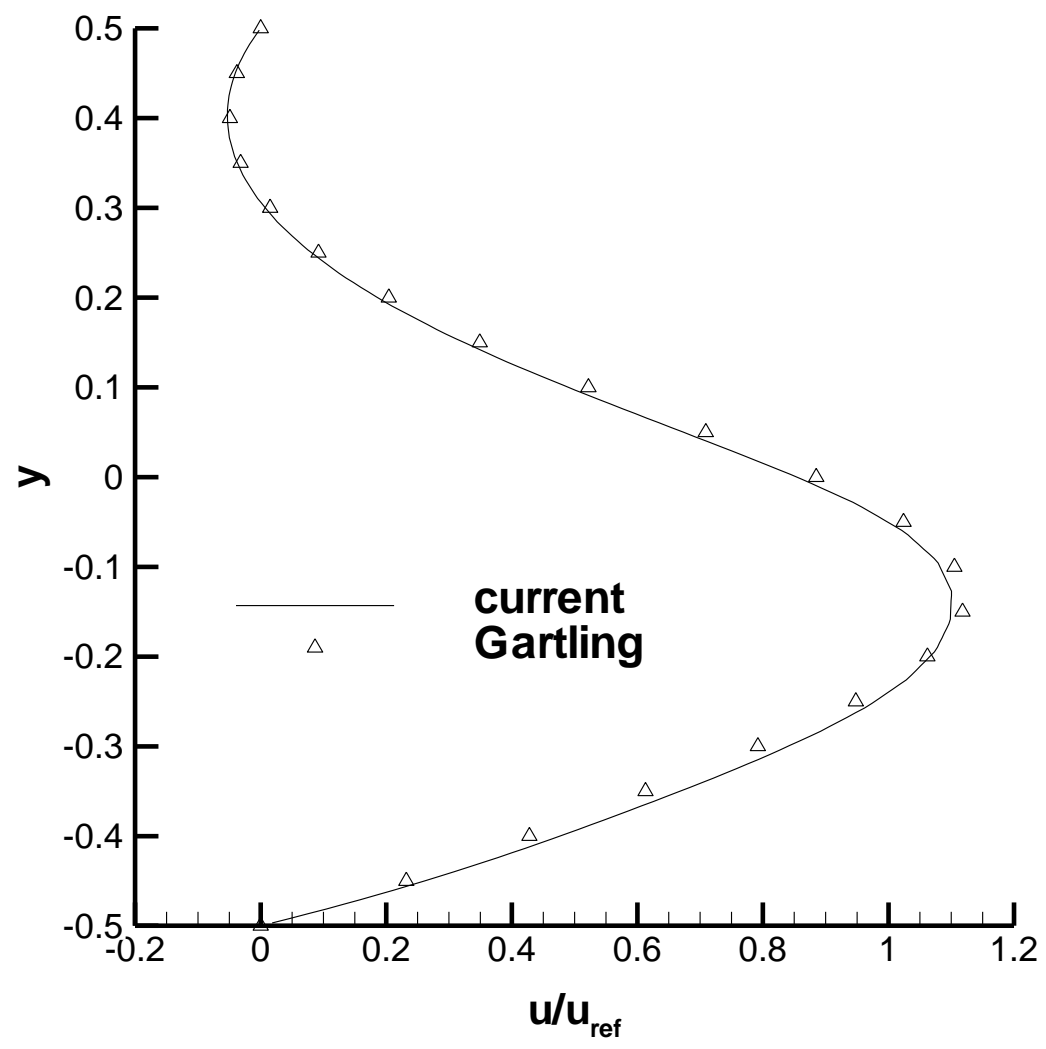

Fig. 8. Comparison of $\mathbf{u} / \mathbf{u}_{\text {ref }}$ at $x=7$ with Gartling's results at $\mathrm{Re}=800$. 


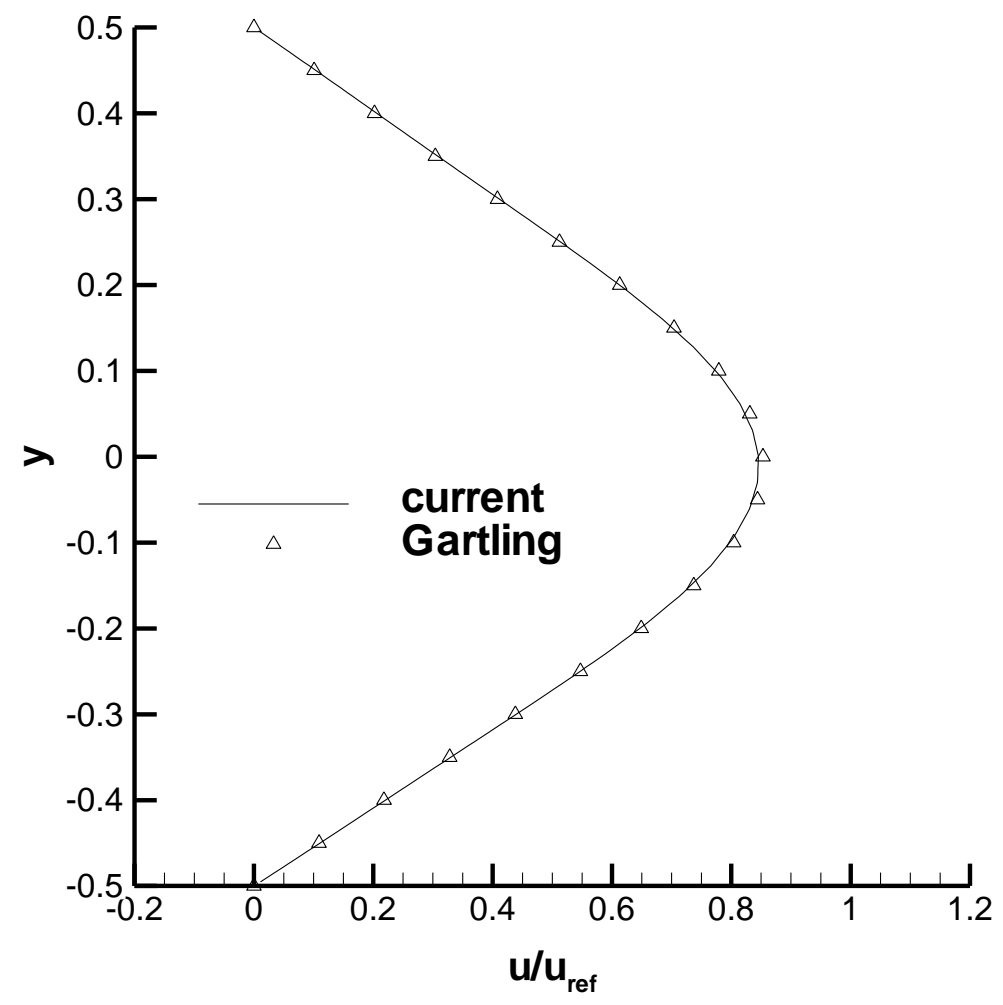

Fig. 9. Comparison of $\mathbf{u} / \mathbf{u}_{\text {ref }}$ at $x=15$ with Gartling's results at $\mathrm{Re}=800$.

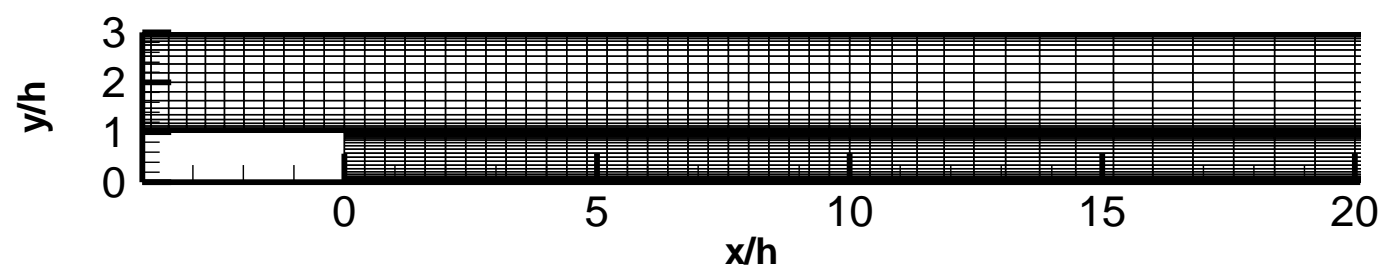

Fig. 10. Grid for turbulent backward-facing step (full grid goes from $\mathrm{x} / \mathrm{h}=-8$ to 30 ) 


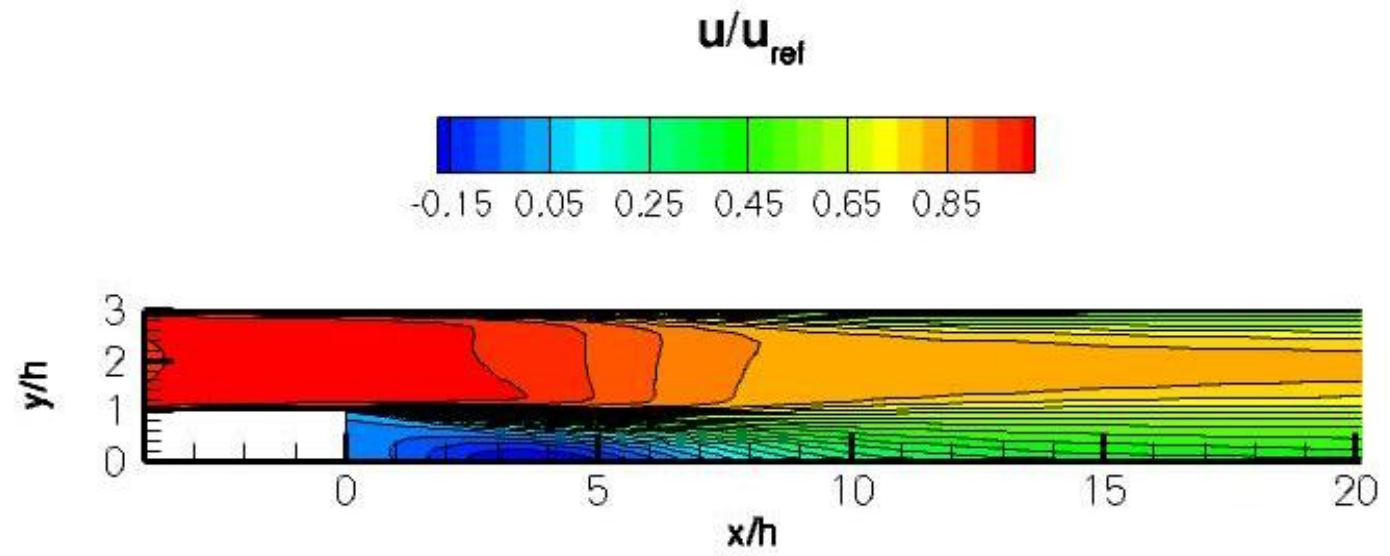

Fig. 11. Contours of $\mathbf{u} / \mathbf{u}_{\text {ref }}$ for turbulent backward-facing step

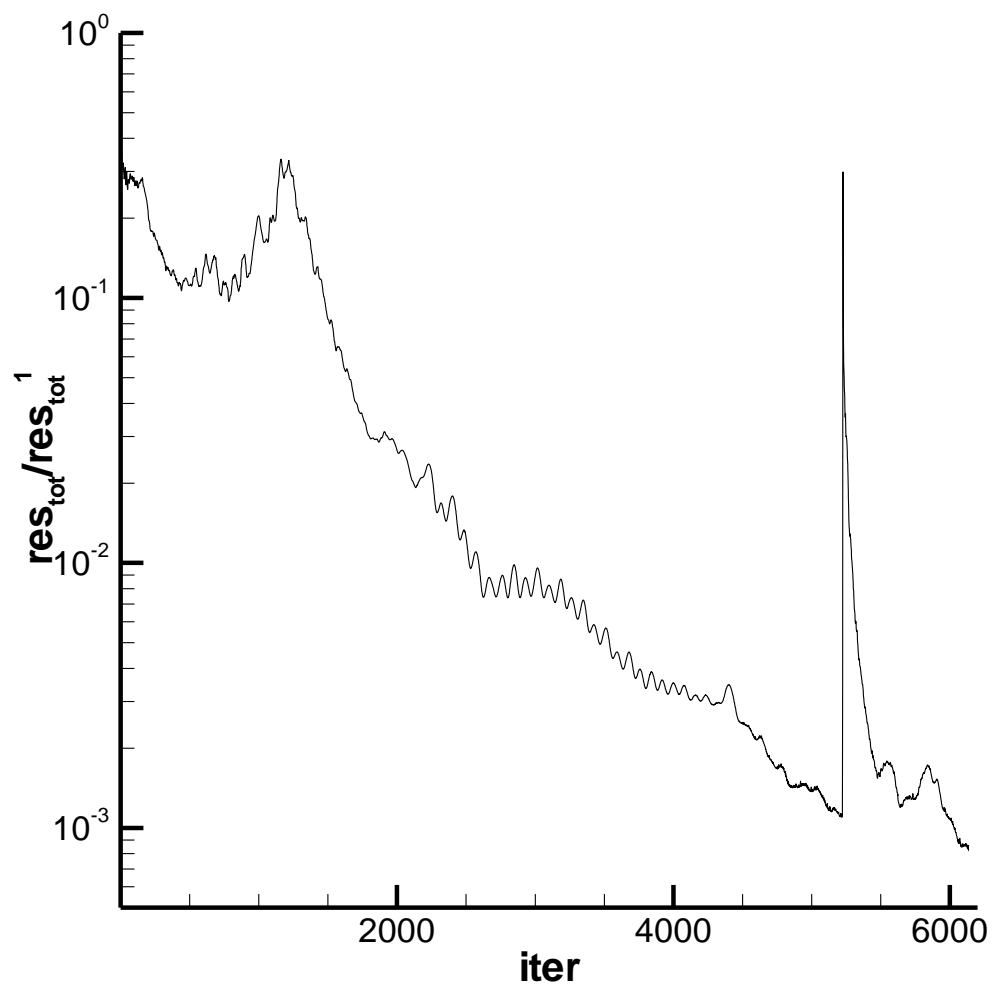

Fig. 12. Convergence of $\mathbf{r e s}_{\text {tot }} / \mathbf{r e s}_{\text {tot }}{ }^{1}$ for the turbulent backward-facing step. 


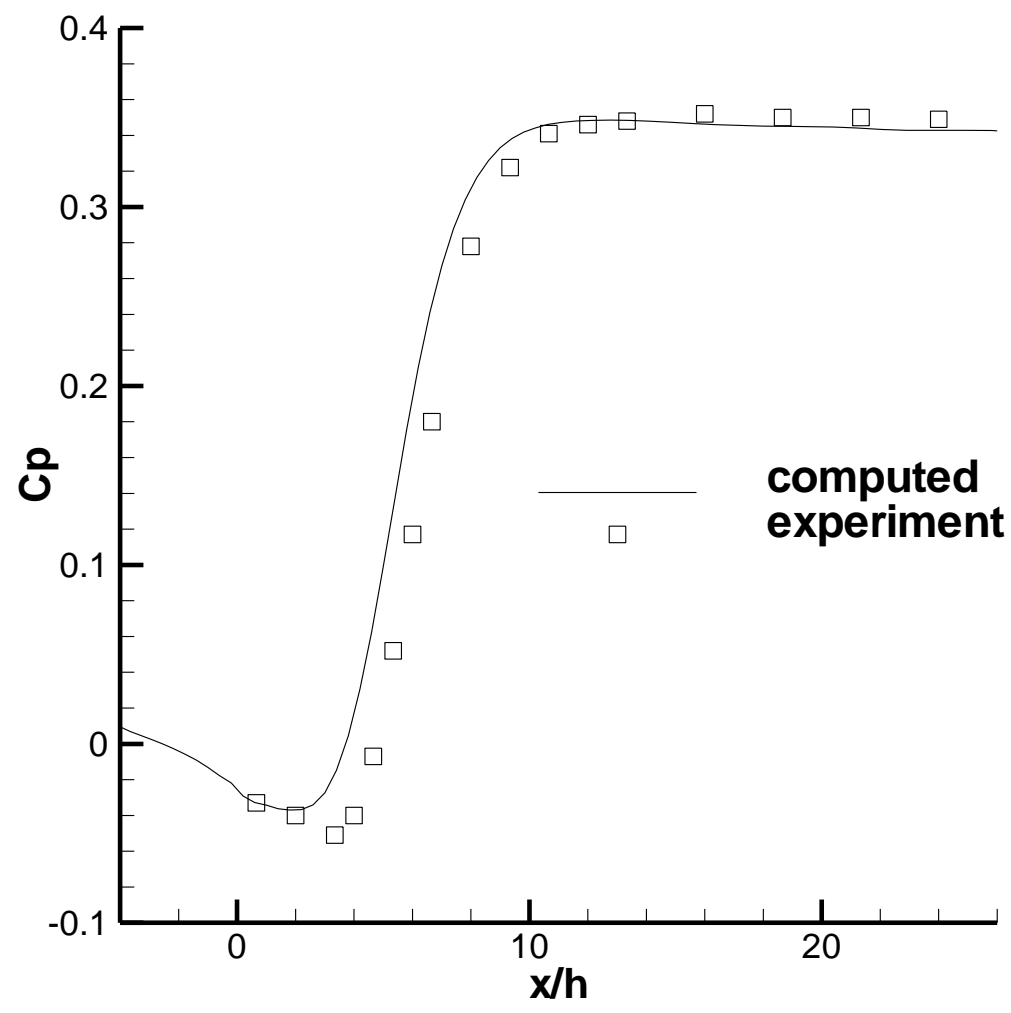

Fig. 13. Comparison of computed and experimental pressure coefficients.

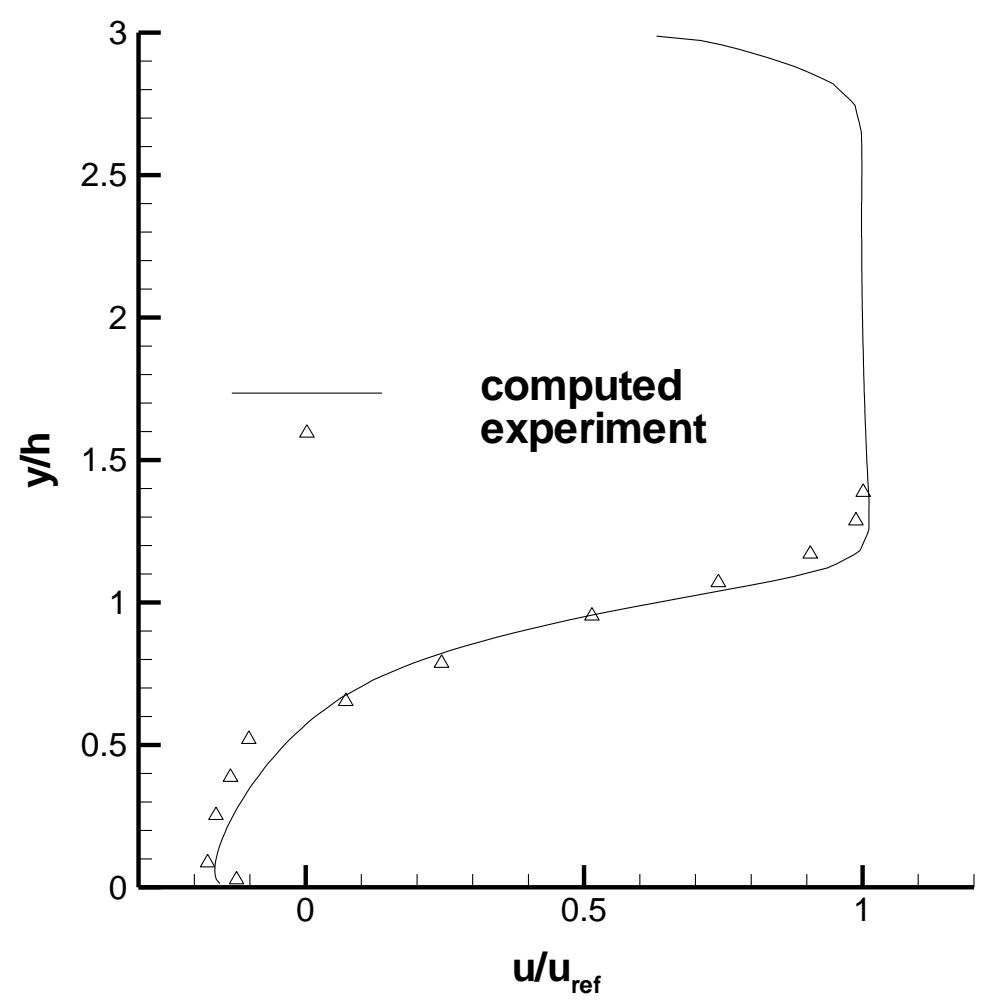

Fig. 14. Comparison of computed $\mathbf{u} / \mathbf{u}_{\text {ref }}$ with data at $\mathbf{x} / \mathbf{h}=2.667$. 


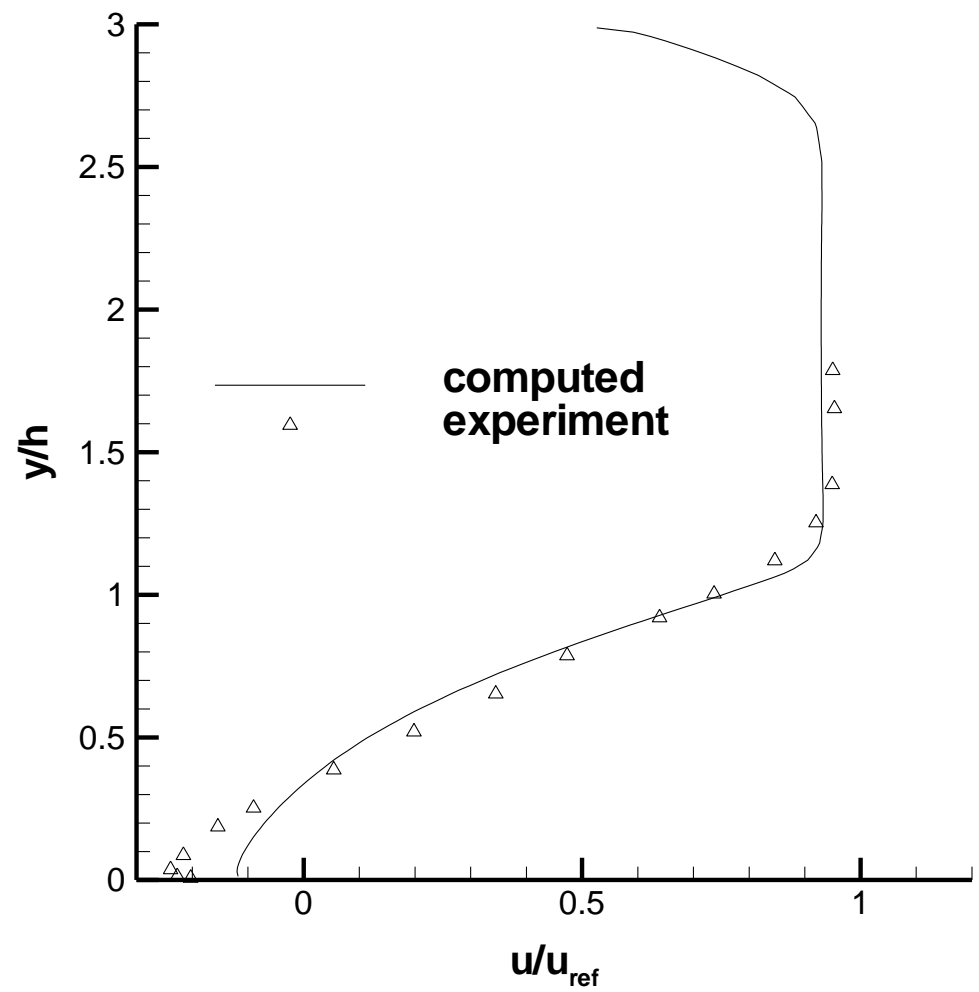

Fig. 15. Comparison of computed $\mathbf{u} / \mathbf{u}_{\text {ref }}$ with data at $\mathbf{x} / \mathbf{h}=5.333$.

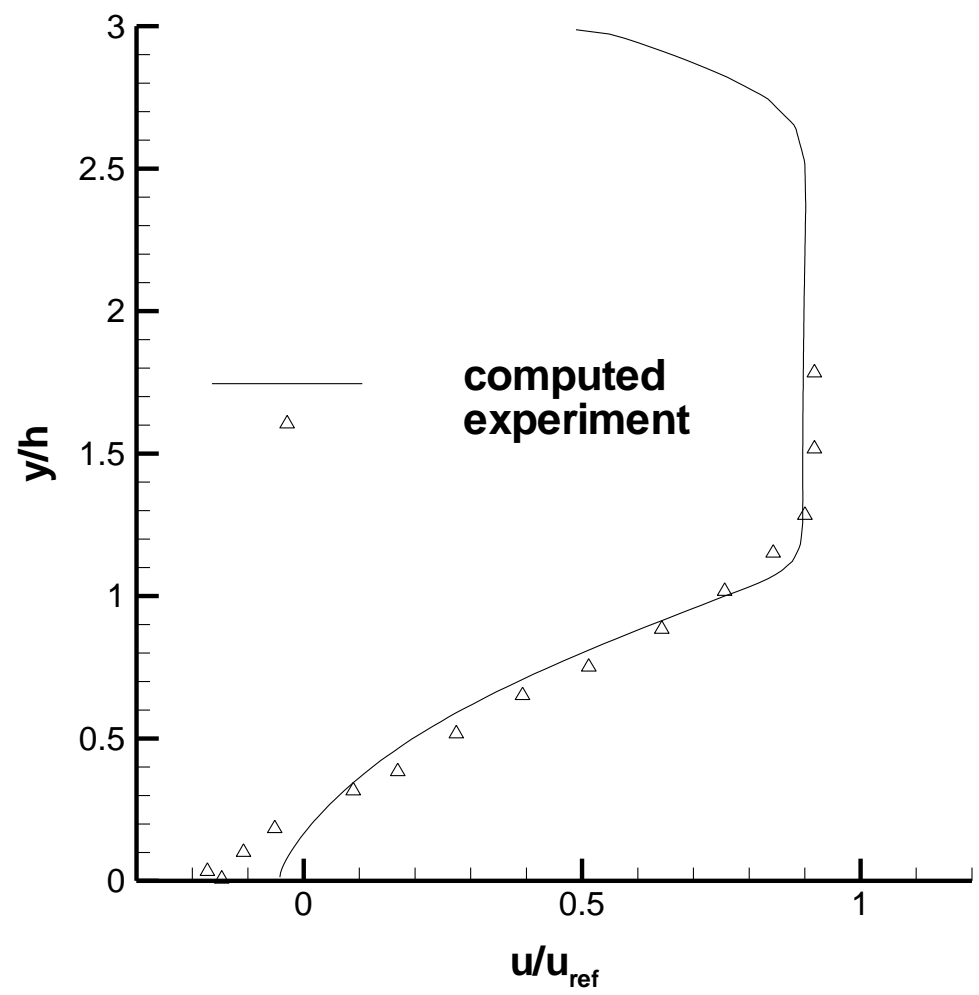

Fig. 16. Comparison of computed $\mathbf{u} / \mathbf{u}_{\text {ref }}$ with data at $\mathbf{x} / \mathbf{h}=6.222$. 


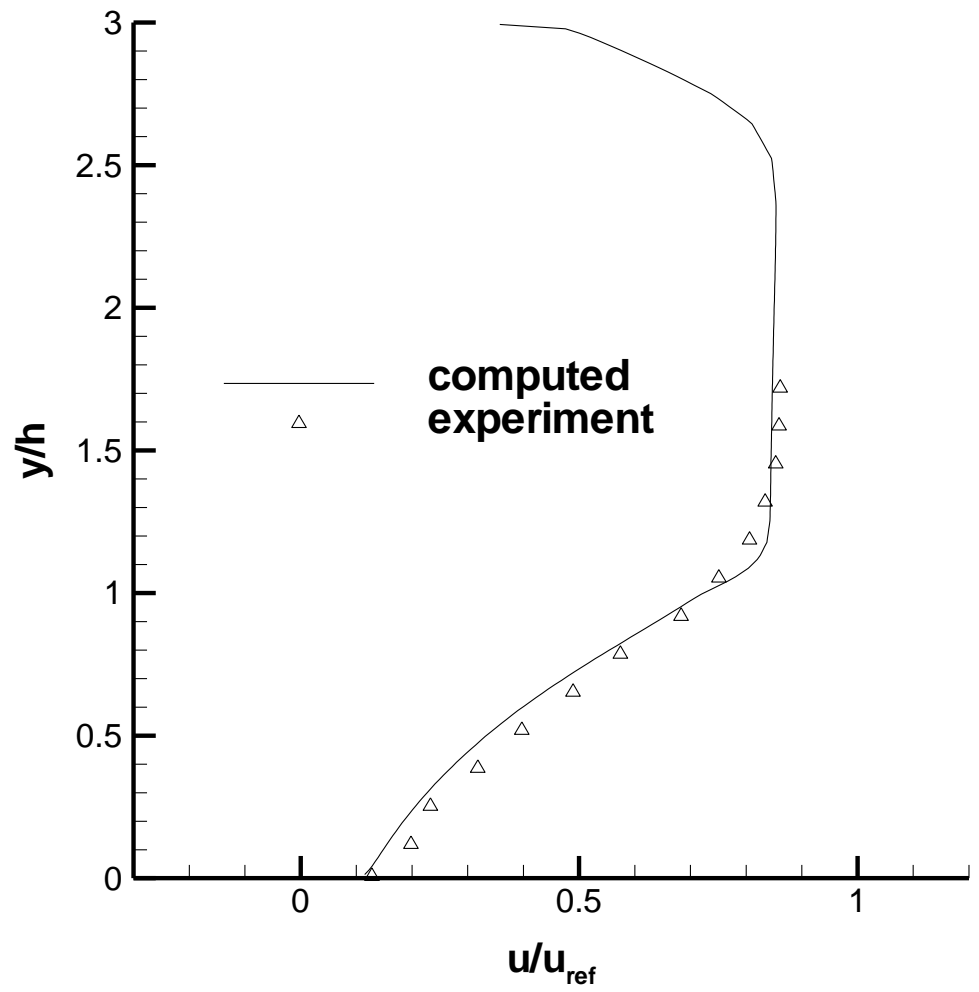

Fig. 17. Comparison of computed $\mathbf{u} / \mathbf{u}_{\text {ref }}$ with data at $\mathbf{x} / \mathbf{h}=8.000$. 